\title{
CARTAS AL EDITOR PUBLICADAS EN REVISTAS BIOMÉDICAS PERUANAS INDIZADAS EN SCIELO-PERÚ 2006-2013
}

\author{
Juan José Montenegro-Idrogo ${ }^{1,2, a}$, Jhon William Mejía-Dolores ${ }^{1,2, b}$, Joel L. Chalco-Huamán ${ }^{1,2, b}$
}

\begin{abstract}
RESUMEN
Estudio bibliométrico que describe las características de cartas al editor publicadas en el período 2006-2013 en revistas biomédicas indizadas a SciELO-Perú. Se recopilaron 253 cartas (10,3\% del total de publicaciones), el mayor número de cartas (139) fue de la Revista Peruana de Medicina Experimental y Salud Pública, con notorio aumento por años. El 25\% de cartas presentaron participación médico-estudiantil. El 14\% de cartas presentaron autores con filiación internacional y $27 \%$ de cartas con filiación endogénica - frecuente en revistas universitarias (AFM, RMH). Los criterios habituales que justifican la publicación de cartas fueron: opinión sobre hecho médico o de dominio público (35,6\%) y discusión de resultados, defectos metodológicos o de interpretación (22,9\%). En revistas biomédicas indizadas a SciELO-Perú las cartas al editor constituyen un porcentaje de publicaciones que ha aumentado en los últimos años, con baja la publicación de cartas de hallazgos o datos propios, en comparación con criterios de opinión o crítica.
\end{abstract}

Palabras clave: Publicaciones científicas y técnicas; Investigación biomédica; Publicaciones (fuente DeCS BIREME).

\section{LETTERS TO THE EDITOR PUBLISHED IN PERUVIAN BIOMEDICAL JOURNALS INDEXED IN SCIELO - PERU 2006-2013}

\begin{abstract}
This bibliometric study describes the characteristics of letters to the editor published between 2006-2013 in biomedical journals indexed in SciELO-Peru.253 letters (10.3\% of total publications) were collected. Most letters (139) were in the Peruvian Journal of Experimental Medicine and Public Health, with marked increase throughout those years. $25 \%$ of letters submitted included medical student participation. 14\% of authors presented with international affiliations and $27 \%$ with endogenous affiliation - common in university journals (Anales de la Facultad de Medicina, Revista Médica Herediana).The usual criteria justifying the publication of letters were: opinion of medical fact or public domain (35.6\%) and discussion of results, methodological flaws or interpretation (22.9\%). In biomedical journals indexed in SciELO Peru the letters to the editor comprise a percentage of publications that has increased in recent years, with low publication of letters of findings or primary data, compared with opinion or criticism.
\end{abstract}

Key words: Scientific and technical publications; Biomedical research; Publications (source: MeSH NLM)

\section{INTRODUCCIÓN}

La carta al editor (letter, correspondence, coment) es una forma de publicación científica breve y específica considerada un medio de interacción que promueve el intercambio de nuevo conocimiento, confrontando, esclareciendo, enriqueciendo o aportando nuevas ideas que actualizan y fortalecen la evidencia de determinados temas, asimismo, están sujetas a un proceso de revisión ${ }^{(1-3)}$.
Por la simplicidad en la estructura, estas publicaciones aparentan ser más factibles de elaborar, sin embargo, su aceptación en una determinada revista es multifactorial, dependiendo de la relevancia y/o novedad que brinda ${ }^{(2-4)}$. Así, no queda exenta de la rigurosidad del proceso de revisión de la revista que garantiza su calidad, además, se comporta como un medio que complementa y/o critica la información pospublicación de artículos científicos ${ }^{(5,6)}$. El International Committee of Medical Journal Editors (ICJME) ${ }^{(7)}$ menciona la importancia de esta sección

\footnotetext{
Facultad de Medicina, Universidad Nacional Mayor de San Marcos. Lima, Perú.

Sociedad Científica San Fernando, Universidad Nacional Mayor de San Marcos. Lima, Perú.

Médico cirujano; ${ }^{\mathrm{b}}$ estudiante de Medicina.

Recibido: 20-06-14 Aprobado: 19-11-14
}

Citar como: Montenegro-Idrogo JJ, Mejía-Dolores JW, Chalco-Huamán JL. Cartas al editor publicadas en revistas biomédicas peruanas indizadas en ScIELoPerú 2006-2013. Rev Peru Med Exp Salud Publica. 2015;32(1):104-9. 
en las revistas científicas, ya que su ausencia niega a los lectores la posibilidad de responder a los artículos publicados (2,3); sin embargo, suele subestimarse o exagerar el rol crítico de este tipo de publicaciones ${ }^{(8,9)}$.

Se menciona que estas publicaciones en idioma español se presentan con menor frecuencia que en la literatura en idioma inglés y, en su mayoría, se refieren más a críticas o comentarios sobre un artículo previo que aportes originales propios ${ }^{(2,10)}$. En Perú, la cultura de publicación científica es diferente y menor en relación a otras realidades ${ }^{(11)}$, solo se cuenta con dos revistas biomédicas peruanas indizadas a Medline, y ninguna revista médica indizada a ISI Web of Science; a pesar que se evidencia un aumento de la producción científica peruana, esta aún no es comparable con otras realidades en estas bases de datos mundiales como se evidencia en SCImago Journal and Country Rank (http://www.scimagojr.com/). Al ser SciELO una plataforma regional, la participación de publicaciones peruanas es más factible, sin embargo, se desconoce el comportamiento de esta forma de publicación y la evolución que ha tenido en los últimos años, del que podría inferir la visibilidad de estas revistas peruanas indizadas como la actividad de la comunidad científica biomédica local.

Así, el estudio tiene por objetivo describir la frecuencia y características de las cartas al editor de las revistas biomédicas peruanas indexadas en la actualidad en la base de datos SciELO-Perú entre los años 2006 y 2013.

\section{EL ESTUDIO}

Estudio descriptivo de tipo bibliométrico de las revistas biomédicas indizadas en la base de datos de SciELOPerú hasta el año 2013.

Se realizó la búsqueda mediante la página oficial de SciELO Perú: www.scielo.org.pe/ (último acceso 3 de mayo de 2014): accediendo a las revistas biomédicas indizadas actualmente y de recuperación electrónica total correspondientes al período 2006-2013 (volúmenes periódicos completos). Luego de la búsqueda se procedió a la recopilación de los archivos correspondientes únicamente a la categoría "Cartas al editor", las cuales fueron archivadas y numeradas según período para la fase de recopilación de información. Las características descritas de las cartas publicadas fueron:

- Año y revista de publicación: correspondiente al período y nombre actual de la revista en la base de datos de SciELO-Perú.
- Filiación: correspondiente al nombre de la institución de la que forma parte el autor mencionado en la publicación. Se consideraron las instituciones citadas como filiación por el autor, las que no presentaron filiación se consideró como tal. Se consideró, además, la categoría académica consignada por los autores en la publicación (solo estudiantil, solo profesionales médicos, estudiantil y profesionales médicos, otros). Se consignó también el comportamiento de la filiación según pertenencia de los autores a la institución de la revista: 1) endogénica: autores pertenecientes a la misma institución de la revista; 2) filiación internacional: autores pertenecientes a instituciones de otra nacionalidad diferente a la peruana.

- Finalidad o justificación para publicar la carta al editor. Se utilizaron los criterios de Velazquez- Jones (descritos por Miyahira) ${ }^{(12)}$ al que se estructuró en orden sistemático en tres ejes principales y uno no especificado (Tabla 1). Los criterios indican el motivo fundamental que justifica la publicación: 1) eje 1 (opinión o juicio) carta que desarrolla su tema sin presentar hallazgos propios, (eje 3) ni en relación a un artículo publicado (eje 2); 2) eje 2 (en relación a un trabajo recientemente publicado), parte de un artículo previo publicado que critica o complementa; 3) eje 3 (hallazgos propios) presenta datos generados de manera original y replicable y/o reportable. En este caso los criterios se consideraron excluyentes.

Tabla 1. Criterios que justifican la publicación de cartas al editor*

\begin{tabular}{cl}
\hline \multicolumn{2}{c}{ Ejes de justificación } \\
\hline 1. Opinión o juicio & $\begin{array}{l}\text { 1.1. Sobre política editorial } \\
\text { 1.2. Sobre hecho médico o de domi- } \\
\text { nio público }\end{array}$ \\
$\begin{array}{l}\text { 2.1. Ampliar, interpretar o explicar } \\
\text { algunos aspectos }\end{array}$ \\
$\begin{array}{ll}\text { 2. Trabajo de inves- } \\
\text { mente publicado }\end{array}$ & $\begin{array}{l}\text { 2.2. Discutir resultados, señalar defec- } \\
\text { tos metodológicos o de interpretación } \\
\text { de resultados }\end{array}$ \\
$\begin{array}{l}\text { 3.1. Resultados de estudio semejante } \\
\text { a otro publicado en la revista }\end{array}$ \\
$\begin{array}{l}\text { 3.2. Hallazgo clínico o experimental } \\
\text { no descrito previamente } \\
\text { 3.3. Investigaciones que no se publi- } \\
\text { carían como artículo original } \\
\text { Comentario que no aporta mayor } \\
\text { información (excluye criterio 1) }\end{array}$ \\
\hline
\end{tabular}

\footnotetext{
* Modificado de Velazquez- Jones.
} 
Se realizó el respectivo análisis descriptivo según año, revista y las variables descritas usando frecuencias y porcentajes mediante tablas y gráficos de barras. La información utilizada es de acceso público y al ser un estudio con fuentes de datos bibliográficas no fue necesaria la aprobación por un comité de ética.

\section{HALLAZGOS}

Las revistas biomédicas peruanas indizadas hasta el 2013 a SciELO Perú son seis: Acta Médica Peruana (AMP); Anales de la Facultad de Medicina (AFM); Revista Médica Herediana (RMH); Revista Peruana de Ginecología y Obstetricia (RPGO); Revista Peruana de Gastroenterología (RPG) y Revista Peruana de Medicina Experimental y Salud Pública (RPMESP); estas dos últimas indizadas a Medline.

En el período 2006-2013 el total de cartas al editor publicadas en las revistas biomédicas peruanas indizadas a SciELO fue de $253,10,3 \%$ del total de publicaciones de estas revistas en el período de estudio. El porcentaje de publicaciones que corresponden a cartas para cada revista fue: RPMESP 139 cartas (17,1\%); RMH 52 cartas (14,6\%); AMP 21 cartas $(5,6 \%)$, RPG 21 cartas (5,1\%); AFM 18 cartas $(4,5 \%)$, y RPGO 2 cartas $(1,9 \%)$. Se evidencia un aumento global en el número de cartas publicadas según año, pero la distribución porcentual de cartas por años fue irregular para cada revista, excepto en la RPMESP donde se evidencia aumento en el número de cartas por año a partir del año 2008 (Figura 1A).

La participación médico-estudiantil en la autoría de cartas fue de $25,7 \%$, con una distribución irregular según años, con dos picos: uno durante los años 20072008 entre 20 y $30 \%$ de las cartas publicadas en esos años y otro en los años 2011-2013 entre 25 y 40\% de las cartas publicadas para dicho período (Figura 1B).

Se encontró $68(26,9 \%)$ cartas con filiación endogénica y $36(14,2 \%)$ cartas con filiación de institución internacional. En el caso de cartas con filiación endogénica las más frecuentes fueron AFM $(68,2 \%)$ y RMH $(53,8 \%)$. La RPMESP presentó mayor porcentaje de cartas con filiación internacional 29 (20,9\%) (Tabla 2).

En este período se encontraron 22 cartas de réplica única y 2 casos de continuación en la comunicación referente a un tema (1. Controversias respecto del uso del timerosal: 4 cartas en AFM, 2 cartas en AMP; 2 . Controversias sobre aprobación de posible ensayo clínico). Se encontró además 9 impertinentes con los criterios de carta al editor.
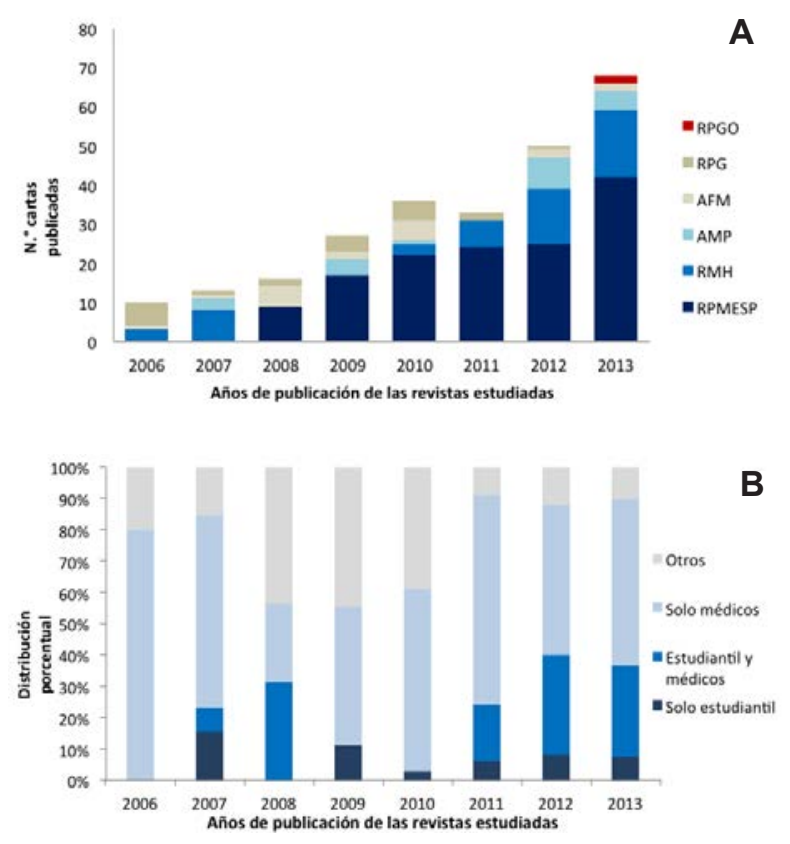

Figura 1. A. Cartas al editor publicadas en revistas biomédicas indizadas a SciELO-Perú 2006-2013, según revista y año de publicación. B. Cartas al editor publicadas en revistas biomédicas indizadas a SciELO-Perú 2006-2013, según categorías académicas de autores y año de publicación.

RPMESP: Rev. Per. Med. Exp. Salud Pública; RPG: Revista Peruana de Gastroenterología; RMH: Revista Médica Herediana; AFM: Anales de la Facultad de Medicina; AMP: Acta Médica Peruana.

El criterio de publicación más frecuente de las cartas fue "Sobre un hecho médico o de dominio público" 90 cartas $(35,6 \%)$, "Discusión de resultados o defectos metodológicos" 58 cartas (22,9\%), e "Investigaciones que no se publicaron como artículo original" 48 cartas $(19,0 \%)$. El comportamiento porcentual fue similar por revistas (Tabla 3).

Tabla 2. Frecuencia de Cartas al editor publicadas en revistas biomédicas peruanas indizadas a SciELO Perú 2006-2013, según filiación endogénica e internacional

\begin{tabular}{lcccc}
\hline \multirow{2}{*}{ Revista (n) } & \multicolumn{4}{c}{ Tipo de filiación } \\
\cline { 2 - 5 } & Endogénica & $\mathbf{( \% )}$ & Internacional & $\mathbf{( \% )}$ \\
\hline RPMESP (139) & 23 & $(16,5)$ & 29 & $(20,9)$ \\
\hline RMH (52) & 28 & $(53,8)$ & 3 & $(5,8)$ \\
AFM (22) & 15 & $(68,2)$ & 3 & $(13,6)$ \\
AMP (21) & 2 & $(9,5)$ & 0 & $(0)$ \\
RPG (17) & 0 & $(0)$ & 1 & $(5,9)$ \\
RPGO (2) & 0 & $(0)$ & 0 & $(0)$ \\
TOTAL (253) & 68 & $(26,9)$ & 36 & $(14,2)$ \\
\hline
\end{tabular}

AMP: Acta Médica Peruana; RMH: Revista Médica Herediana; AFM: Anales de la Facultad de Medicina; RPG: Revista Peruana de Gastroenterología; RPMESP: Rev. Per. Med. Exp. Salud Pública.

(n): número total de cartas. 
Tabla 3. Distribución de cartas al editor publicadas en revistas biomédicas peruanas indizadas a SciELO Perú 20062013, según criterio de publicación*

\begin{tabular}{|c|c|c|c|c|c|c|c|c|c|c|c|c|c|c|}
\hline \multirow{2}{*}{ Criterio de publicación de carta } & \multicolumn{12}{|c|}{ Revistas biomédicas peruanas indizadas a SciELO } & \multirow[b]{2}{*}{ Total } & \multirow[b]{2}{*}{$(\%)$} \\
\hline & AFM & $(\%)$ & AMP & $(\%)$ & RPMESP & $(\%)$ & RMH & $(\%)$ & RGP & $(\%)$ & RPGO & $(\%)$ & & \\
\hline Sobre política editorial & -- & -- & -- & -- & 3 & $(2,2)$ & -- & - & 6 & $(35,3)$ & 2 & $(100)$ & 11 & $(4,4)$ \\
\hline Sobre hecho médico o de d & 5 & $(22,7)$ & 12 & $(57,1)$ & 48 & $(34,5)$ & 21 & $(40,4)$ & 4 & $(23,5)$ & -- & -- & 90 & $(35,6)$ \\
\hline Ampliar, interpretar o explicar algunos aspectos & 2 & $(9,1)$ & 3 & $(14,3)$ & 10 & $(7,2)$ & 6 & $(11,5)$ & - & -- & - & - & 21 & $(8,3)$ \\
\hline $\begin{array}{l}\text { Discutir resultados, señalar d } \\
\text { cos o de interpretación de res }\end{array}$ & 7 & $31,8)$ & 4 & $(19,0)$ & 28 & $(20,1)$ & 15 & 28,8) & 4 & 23,5) & -- & -- & 58 & $(22,9)$ \\
\hline $\begin{array}{l}\text { Resultados de estudio semejante a otro } \\
\text { do en la revista }\end{array}$ & 3 & $(13,6)$ & 1 & $(4,8)$ & 3 & $(2,2)$ & 1 & $(1,9)$ & -- & -- & -- & -- & 8 & $(3,2)$ \\
\hline $\begin{array}{l}\text { Hallazgo clínico o experimental no descrito pre- } \\
\text { viamente }\end{array}$ & -- & -- & -- & -- & 7 & $(5,0)$ & 7 & $(13,5)$ & 1 & $(5,9)$ & - & -- & 15 & $(5,9)$ \\
\hline $\begin{array}{l}\text { Investigaciones que no se publicarían como ar- } \\
\text { tículo original }\end{array}$ & 4 & $(18,2)$ & 1 & & 40 & $(28,8)$ & 2 & $(3,8)$ & 1 & $(5,9)$ & - & - & 48 & $(19,0)$ \\
\hline $\begin{array}{l}\text { Comentario que no aporta mayor información } \\
\text { (excluye criterio 1) }\end{array}$ & 1 & $(4,5)$ & -- & -- & -- & -- & -- & -- & 1 & $(5,9)$ & - & -- & 2 & $(0,8)$ \\
\hline
\end{tabular}

AMP: Acta Médica Peruana; RMH: Revista Médica Herediana; AFM: Anales de la Facultad de Medicina ; RGP: Revista de Gastroenterología del Perú; RPMESP: Revista Peruana de Medicina Experimental y Salud Publica; RPGO: Revista Peruana de Ginecología y Obstetricia.

* Modificado de Velazquez-Jones.

\section{DISCUSIÓN}

La frecuencia de cartas al editor en revistas peruanas indizadas a SciELO Perú fue de 253 cartas $(10,3 \%$ del total de publicaciones). En las revistas peruanas en la década pasada, la publicación de cartas presenta un comportamiento irregular, incluso con revistas que no cuentan con esta sección, lo que contrasta la brecha existente con revistas científicas de países del primer mundo ${ }^{(13)}$. Huamani $\mathrm{C}$, describe dentro de la producción científica de las revistas biomédicas peruanas un total de 1053 publicaciones con solo un $3,5 \%$ de cartas al editor en el período 2005-2007 (14). En otro estudio de producción científica peruana en revistas indizadas en ISI Web of Science 2000-2009, el porcentaje de cartas al editor fue de $7,4 \%{ }^{(15)}$.

El presente estudio evidencia aumento de la frecuencia de publicación de cartas al editor a partir del año 2008, con predominio de la RPMESP. Cabe recordar que en este período se da la transición a la indexación adicional de esta revista a la base de datos Medline. En la literatura científica latinoamericana la publicación de cartas es menor por número de publicación ${ }^{(1,10)}$. En la literatura científica internacional se ha reportado comportamiento constante de publicación de cartas al editor en revistas de alto impacto, así, Annals of Internal Medicine, New England Journal of Medicine, The American Journal of Medicine o Lancet, publican entre 8 y 10 cartas por número; en la Revista Médica de Chile durante el 2012, 13,6 \% de las publicaciones correspondieron a cartas al editor ${ }^{(16)}$, mayor porcentaje que el reportado en revistas peruanas; cabe señalar que las indexaciones adicionales a bases de datos como ISI Web of Science de esta revista, además del mayor impacto en relación a otras revistas latinoamericanas como las peruanas. Así, la participación de cartas puede considerarse multifactorial, dependiendo del impacto o visibilidad de la revista, así como la cultura de publicación de la comunidad científica a la que se encuentra circunscrita.

La distribución irregular por años de cartas al editor que encontramos en el presente estudio suele presentarse en revistas biomédicas latinoamericanas y la periodicidad de las mismas representa un factor clave. Las cartas pertenecientes a réplica son consideradas pertinentes; sin embargo, también se presentaron casos de respuesta múltiple. Donde a pesar de ser temas de opinión o apreciación personal, no se deben excluir de un adecuado proceso editorial ${ }^{(5,6)}$. Gotzche evidencia, además, que existe una respuesta negativa ante las críticas reportadas mediante cartas por parte de los autores, así los editores deben asegurarse que los autores respondan adecuadamente las críticas planteadas a su artículo ${ }^{(17,18)}$.

La filiación endogénica se presentó en más de la cuarta parte de las cartas al editor. Se evidencia que más de la mitad de las cartas de las revistas universitarias (RMH $53,8 \%$ y AFM $68,2 \%$ ) tuvieron filiación endogénica, lo que evidencia visibilidad local por parte de estas revistas, sin embargo, el porcentaje de cartas con filiación internacional es menor del $15 \%$ y solo la RPMESP conforma el $21 \%$ de cartas con filiación internacional, debido probablemente a su ingreso en dicho periodo a la base de datos Medline, a diferencia de las demás revistas, comportamiento que no es acorde con la RPG quien está indexada a Medline desde años anteriores, lo que implica una visibilidad predominantemente local, y el comportamiento de publicación dependerá de este público. 
En la autoría, la participación médico estudiantil en cartas al editor fue de $25,7 \%$ con incremento a partir del año 2008 en el presente estudio. La participación estudiantil en la publicación de cartas también se evidencia de manera incipiente en la revista CIMEL entre los años 2006-2009 (19). Esta tendencia en la publicación biomédica se menciona por Huamaní et al. años anteriores ${ }^{(20)}$, donde se evidencia mayor participación de estudiantes de medicina en la publicación en revistas biomédicas peruanas con $4,5 \%$ del total de publicaciones realizadas en dicho período. Este comportamiento evidencia cambios en los últimos años en la cultura de publicación biomédica desde el pregrado.

La frecuencia de cartas al editor que aportan datos originales es menos del $30 \%$, y menos del $20 \%$ tuvo como motivo una investigación que no fue publicada como original, mientras que las cartas al editor más frecuentes son aquellas relacionadas a comentar un hecho o criticar un artículo previo. Este comportamiento es similar al mencionado para revistas en idioma español ${ }^{(1,10)}$, aunque se destaca la importancia de la crítica y debate en las cartas ${ }^{(2,6,7)}$, la finalidad de las publicaciones científicas en general debe aportar información propia e inédita, que no está exenta de esta forma de publicación ${ }^{(1,10)}$, que no es característica de la participación predominantemente local con una cultura de publicación aún no comparable con países con alto índice de publicación donde se evidencia mayor aporte de datos originales que críticas a una publicación previa, como ocurre en Latinoamérica ${ }^{(10,11)}$.
Así, se consideraría que en años previos la cultura de publicación de cartas en revistas peruanas era inmadura y escasa, que se refleja en el número de cartas, la participación estudiantil cada vez mayor, y la menor filiación endogénica y filiación internacional, debido también por una escasa visibilidad que se refleja en las indizaciones predominantemente regionales de estas revistas, que determinará un público mayoritariamente local y la participación de este dependerá de la cultura de publicación que tengan.

Se puede concluir que la frecuencia de publicación de cartas al editor en revistas biomédicas indexadas a SciELO-Perú ha aumentado según años, sin embargo, no es regular en todas estas revistas y el porcentaje de filiación internacional de estas es menos de la cuarta parte. Es importante el aporte médicoestudiantil descrito de cerca de la cuarta parte de cartas publicadas. Es necesario reforzar el proceso de revisión y promover esta forma de publicación por parte de estas revistas biomédicas, además de promover la cultura de publicación en la comunidad biomédica peruana que garantice la producción y el flujo de información de calidad en estas y otras revistas científicas.

Contribuciones de autoría: MIJJ realizó la concepción del artículo, obtención de resultados, análisis de datos, redacción del artículo, revisión crítica del artículo y su aprobación final. MDJW y ChHJL participaron en la obtención de resultados, redacción del artículo y la aprobación de la versión final.

\section{Fuentes de financiamiento: autofinanciado.}

Conflictos de interés: los autores declaran no tener conflictos de interés.

\section{REFERENCIAS BIBLIOGRÁFICAS}

1. Johnson C, Green B. Helpful hints: writing effective letters to the editor. J Manip Physiol Ther. 2006;29(6): 415-6.

2. Bhopal RS, Tonks A. The role of letters in reviewing research. BMJ. 1994 Jun 18;308(6944):1582-3.

3. Winker MA, Fontanarosa PB. Letters: a forum for scientific discourse. JAMA. 1999 Apr 28;281(16): 1543 .

4. Ortinau D. Writing and publishing important scientific articles: A reviewer's perspective. J Bus Res. 2011;64:150-6.

5. Horton R. Postpublication criticism and the shaping of clinical knowledge. JAMA. 2002 Jun 5;287(21):2843-7.

6. Overton DT, Moorhead JC. Letters, we've got letters.... Ann Emerg Med.
2009 Nov;54(5):751-2. doi: 10.1016/j. annemergmed.2009.05.026.

7. International Committee of Medical Journal Editors. Uniform requirements for manuscripts submitted to biomedical journals [Internet]. ICMJE; 1997 [citado el 12 de enero del 2010]. Disponible en: http://www.icmje.org

8. Yang C, Srinivasan P, Polgreen P. Automatic adverse drug events detection using letters to the editor. AMIA Annu Symp Proc. 2012;2012:1030-9.

9. Goodman NW. How to write a critical letter and respond to one. Hosp Med. 2001 Jul;62(79:426-7.

10. Brennan PA1, Mitchell DA, Walker TW, Cascarini L, Oeppen RS. Short communication articles published in BJOMS during 2008-2009--an analysis of types of submission and subject material. Br J Oral Maxillofac Surg. 2010 Apr;48(3):167-72. doi: 10.1016/j.bjoms.2010.01.008.

11. Montenegro-Idrogo JJ, HuertaCollado YM. Cultura de publicación: realidades diferentes. Rev Med Chile. 2014;142:402.

12. Miyahira J. Importancia de las cartas al editor. Rev Med Hered. 2010;21(2):57-8.

13. Pamo O. Estado actual de las publicaciones periódicas científicas del Perú. Rev Med Hered 2004;16(1):65-73.

14. Huamani C, Pacheco-Romero J. Visibilidad y producción de las 
revistas biomédicas peruanas. Rev. Gastroenterol Peru. 2009;29(2):132-9.

15. Huamaní C, Mayta-Tristán P. Producción científica peruana en medicina y redes de colaboración, análisis del Science Citation Index 2000-2009. Rev Peru Med Exp Salud Publica. 2010;27(3):315-25.

16. Reyes H, Bustos-González A, Andresen M, Palma J. La Revista Médica de Chile en el año 2012. Rev Med Chile. 2013;141(8):1049-56.

17. Kleis R. Participation through letters to the editor: Circulation, considerations, and genres in the letters institution. Journalism. 2010;11(1):21-35.

18. Gotzsche P, Delamothe T, Godlee F, Lundh A. Adequacy of authors' replies to criticism raised in electronic letters to the editor: cohort study. BMJ 2010;341:c3926.

19. Montenegro-Idrogo JJ. Características de las Cartas al Editor publicadas en la Revista CIMEL 2005-2009. CIMEL. 2010;15(2):95-6.

20. Huamaní C, Chávez-Solis P, MaytaTristán P. Aporte estudiantil en la publicación de artículos científicos en revistas médicas indizadas en SciELOPerú, 1997 - 2005. An Fac Med. 2008;69(1):42-5.

Correspondencia: Juan José Montenegro Idrogo

Dirección: Av. Arnaldo Márquez 579, Interior 507, Jesús María, Lima, Perú. Teléfono: (+51) 961072186

Correoelectrónico:montenegroidrogo@ gmail.com

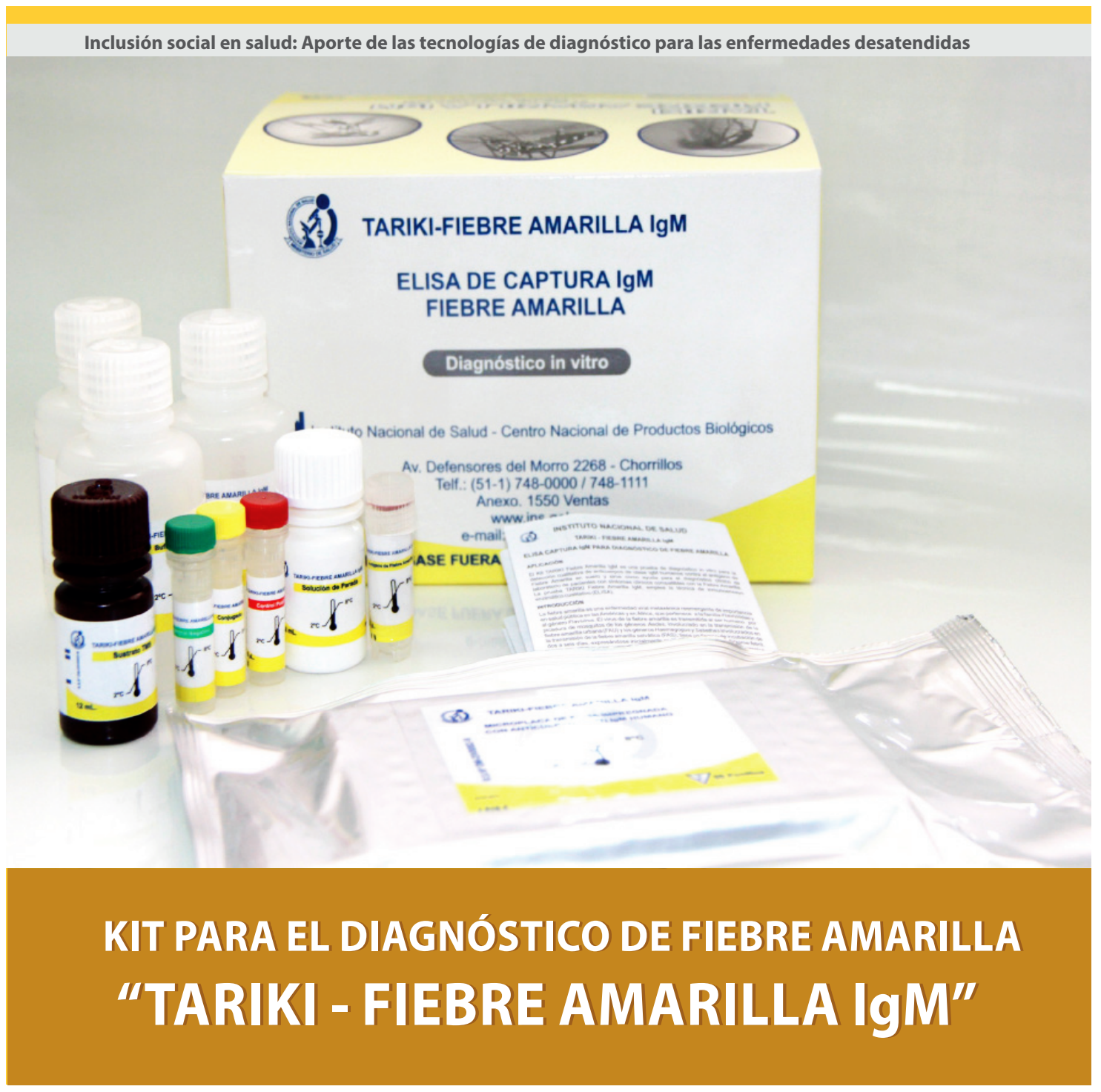

